\title{
Interpretation and Modeling of Laser-Induced Backside Wet Etching Procedure
}

\author{
Csaba VASS $^{* 1}$, Judit BUDAI ${ }^{* 1}$, Zoltán SCHAY $^{* 2}$ and Béla HOPP ${ }^{* 3}$ \\ ${ }^{* 1}$ Department of Optics and Quantum Electronics, University of Szeged, \\ H-6720 Szeged, Dóm tér 9, Hungary \\ E-mail:vasscsaba@physx.u-szeged.hu \\ ${ }^{* 2}$ Department of Surface Chemistry and Catalysis, Institute of Isotopes, \\ 1121 Budapest, Konkoly Thege M. út 29-33, Hungary \\ ${ }^{* 3}$ Research Group on Laser Physics of the Hungarian Academy of Sciences, \\ H-6720 Szeged, Dóm tér 9, Hungary
}

\begin{abstract}
Laser-induced backside wet etched fused silica surfaces were characterized by X-ray photoelectron spectroscopy (XPS) and ellipsomerty, and a new numerical model was developed to interpret the etching procedure on the basis of our recent results. ArF and KrF lasers were used for etching, while the applied liquid absorbers were naphthalene/methyl-methacrylate and pyrene/acetone solutions. The XPS measurements showed that the completely cleaned, etched fused silica surface layers were contaminated by carbon (which originated from the organic absorber molecules). The optical parameters of the modified layers were measured by spectroscopic ellipsometer. The thicknesses of these carbon contaminated layers were very thin (between 10 and $30 \mathrm{~nm}$ ), while their absorption coefficient and the refractive index at $248 \mathrm{~nm}$ were between 100000 and $180000 \mathrm{~cm}^{-1}$, and 1.85 , respectively. Our previous numerical model was completed on the basis of the determined properties of this modified layer, and our results proved that this layer plays key role in the etching procedure. DOI: $10.2961 / \mathrm{jlmn} .2010 .01 .0010$
\end{abstract}

Keywords: laser-induced backside wet etching, carbon contamination, modified layer, XPS spectroscopy, ellipsomerty, numerical model

\section{Introduction}

Dielectrics materials transparent in a wide wavelength range (fused silica, quartz, sapphire, $\mathrm{MgF}_{2}, \mathrm{CaF}_{2}, \mathrm{BaF}_{2}$, etc.) have several application possibilities in refractive and diffractive microoptics and in microfluidics. These materials can be microstructured by conventional techniques which are based on masking procedure (multistep methods): hidrofluidic etching [1], powder blasting [2-3] and ion etching technologies (inductively coupled plasma etching - ICP [4] and reactive ion etching - RIE [5]), and laserbased methods. Hidrofluidic etching and powder blasting have limited resolution and the machined surface is not smooth enough for many optical applications. RIE can reach submicrometer resolution and smooth processed surface, but it is a very complicated and expensive procedure. The laser based techniques can be divided into direct and indirect techniques. In the direct methods infrared and far ultraviolet lasers $\left(\mathrm{CO}_{2}\right.$ [6], $\mathrm{F}_{2}$ [7-8]), soft X-ray beam [9] and femtosecond pulses $[8,10]$ can be used for etching. Although the $\mathrm{CO}_{2}$ laser is applied in industrial environments, but the reachable resolution is some ten micrometer (due to its long wavelength $(10.6 \mu \mathrm{m})$ and thermal based material removal), which is not sufficient for microoptical applications. The other direct methods $\left(\mathrm{F}_{2}\right.$ laser, soft X-ray and femtosecond beam etching) are too complicated at present for real industrial applications. The nanosecond UV lasers (mostly excimers) would be suitable for micromachining of UV-transparent materials, but the effectiveness is very low due to the high etching threshold fluence, which originates from their transparency. The indirect methods, the laser-induced backside wet/dry etching LIBWE/LIBDE [11-19], laser etching at a surface adsorbed layer - LESAL [20] and laser induced plasma assisted ablation - LIPAA [21] are mainly based on UV (excimer) lasers, which has already proved its suitability in industrial environments.

One of the most flexible and promising indirect techniques is the laser-induced backside wet etching. The most important advantages of LIBWE are the followings: 1. low etching threshold fluence (a few hundreds $\mathrm{mJ} / \mathrm{cm}^{2}$, while the direct ablation threshold is a few $\mathrm{J} / \mathrm{cm}^{2}$ [22]); 2. crackand debris-free etched and surrounding areas; 3. one-step method (do not need mask preparation on the sample surface); 4. the depth of the etched area can be easily controlled: minimum etch rate can be lower than $3 \mathrm{~nm} /$ pulse; 5 . good etched surface quality; 6. high lateral resolution. In our previous studies it was proved that LIBWE is suitable for production of submicrometer grating structure in fused silica [23-26]: we fabricated $104 \mathrm{~nm}$ period fused silica grating, which has the minimum grating constant produced by laser techniques in fused silica so far [23].

Although LIBWE is intensively studied nowadays, there are some open questions concerning the etching process. The first one is that the fluence - etch rate graphs can be fitted by two straight lines. This behavior was observed by many research groups [13-14, 27-32], but correct, coherent explanation have not existed yet. We have already presented a LIBWE model in our previous papers [15, 27], which gave relatively good results for LIBWE applying ArF laser (nevertheless the calculated threshold fluence 
was higher than the measured one), but this model did not work for $\mathrm{KrF}$ experiments, namely etching was not predicted in the applied fluence range (up to $\approx 2 \mathrm{~J} / \mathrm{cm}^{2}$ ). The main difference between the two laser sources is in their wavelengths and therefore in the significant difference between the absorption coefficients of the absorbing liquid $\left(\alpha^{\mathrm{ArF}}{ }_{193 \mathrm{~nm}} \sim 50000 \mathrm{~cm}^{-1} ; \alpha^{\mathrm{KrF}}{ }_{248 \mathrm{~nm}} \sim 3000 \mathrm{~cm}^{-1}\right)$. This is the second problem which has to be solved: difficulty of this modeling indicated that there should be something which is not involved in our model. These questions motivated our following studies (surface characterization and modeling of the etching procedure) to give a more complex explanation of LIBWE process.

\section{Experimental and results}

\subsection{Etching: particle deposition - qualitative observa- tion}

Fused silica plates were used as targets in our experiments (Suprasil II, Heraeus). Naphthalene/methylmethacrylate (concentrations: $c=0.85$ and $1.71 \mathrm{M}$; $\left.\mathrm{M}=\mathrm{mol} / \mathrm{dm}^{3}\right)$ and pyrene/acetone $(c=0.4 \mathrm{M})$ solutions were used as liquid absorbers. In the experiments we used ArF and $\mathrm{KrF}$ excimer lasers $\left(\lambda_{\mathrm{ArF}}=193 \mathrm{~nm} ; \lambda_{\mathrm{KrF}}=248 \mathrm{~nm}\right.$; $\left.\tau_{F W H M}=30 \mathrm{~ns}\right)$. The intensity distribution of the beam cross section was trapezoid-like shape. The scheme of our experimental setup was presented previously [28].

After the etching procedure the etched fused silica plates were carefully cleaned by pouring acetone directly onto them. After cleaning a dark, thin graphite-like layer was observed on those places, where the local laser fluence value was just below the etching threshold. The thin layer could be easily removed by ultrasonic bath in acetone or by wiping the surface with acetone soaked paper wadding. This layer was characterized by Raman spectroscopy and it was found that this layer contains mostly amorphous carbon particles [32-34]. The detailed description of this layer can be found in Rico Böhme's PhD thesis [35]. The correct determination of the optical properties (absorption, refractive index) of this layer consisting of carbon particles is difficult, because it scatters the light, which strongly influences the results. These carbon particles can originate from the decomposition of the organic molecules by the ultraviolet photons, and probably can contaminate the surface of the etched fused silica [32-35]. Although this contamination may have significant effect on the etching procedure, it was observed only on those areas where the local laser fluence value was just below the etching threshold. This means that the etched area (produced by irradiation having fluence much higher than the threshold value) was free from these particles, and only the near surrounding area was contaminated by carbon. The shape of the graphite-like particles covered area (deformed ellipse-like "ring") corresponded to the beamshape.

The etched, completely cleaned surface after the removal of the slightly bonded, deposited graphite-like layer was characterized by XPS (X-ray photoelectron spectroscopy) and ellipsomerty. The etched and characterized areas were free from the above described carbon particles; only their surrounding areas was covered by the carbon layer, but these were also completely removed before the follow- ing characterization measurements by ultrasonic bath and wiping.

\subsection{XPS measurements}

The XPS measurements were performed on the etched, completely cleaned (after wiping and cleaning in ultrasonic bath) fused silica surfaces (laser: $\mathrm{KrF}$; fluence: 465 $\mathrm{mJ} / \mathrm{cm}^{2}$; number of laser pulses: 1000 ; etched area: 16.76 $\left.\mathrm{mm}^{2}\right)$. Naphthalene/methyl-methacrylate $\quad(c=0.85$ and $1.71 \mathrm{M})$ and pyrene/acetone $(c=0.4 \mathrm{M})$ solutions were used as liquid absorbers. The XP spectra were collected by a KRATOS XSAM 800 machine using Mg radiation with $120 \mathrm{~W}$ power and FAT mode with $40 \mathrm{eV}$ pass energy. The fused silica plates were cut to about $10 \times 10 \mathrm{~mm}$ size pieces and fixed to the sample holder by double sided carbon adhesive tape. The electrostatic charging of the sample was about $2 \mathrm{eV}$. The $\mathrm{C} 1 \mathrm{~s}$ line at $285 \mathrm{eV}$ binding energy was used for charge referencing.

The spectra of the original and the etched surfaces were compared. The main peaks in both the original and the etched surface's spectra are: O 1s; C 1s; Si 2p. The O 1s and $\mathrm{C} 1 \mathrm{~s}$ peaks do not show significant differences in the untreated and treated cases, although the adventitious carbon made difficult to evaluate the carbon peaks. A significant difference appears in the $\mathrm{Si} 2 \mathrm{p}$ peak: a second peak can be observed due to the laser etching below the main $\mathrm{Si}$ $2 \mathrm{p}$ peak, which refers to $\mathrm{Si}-\mathrm{C}$ bounding (Fig. 1).

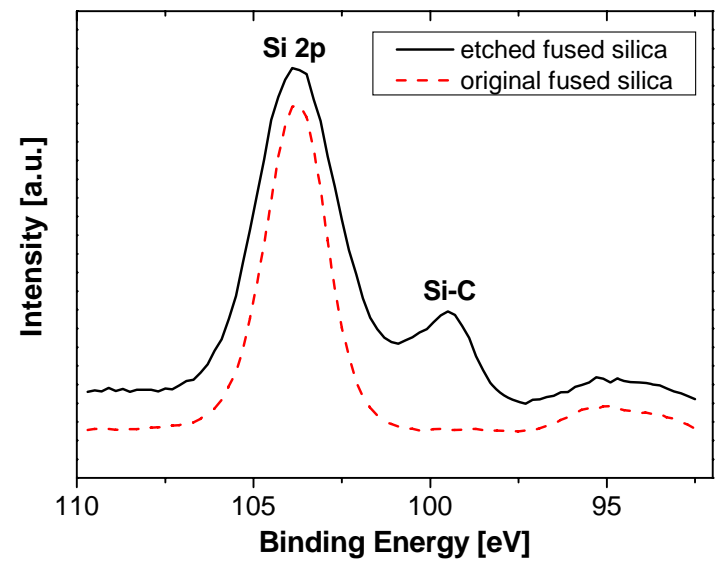

Table 1 The ratio of carbon at different absorbers

\begin{tabular}{lcc}
\hline Liquid absorber & $\alpha\left[\mathrm{cm}^{-1}\right]$ & $\mathrm{Si}-\mathrm{C} / \Sigma \mathrm{Si}[\%]$ \\
\hline Pyrene/acetone 0.4 M & 2100 & 6.75 \\
Naphthalene/methyl-methacrylate $0.85 \mathrm{M}$ & 2830 & 7.7 \\
Naphthalene/methyl-methacrylate $1.71 \mathrm{M}$ & 3380 & 14.4 \\
\hline
\end{tabular}

Fig. 1 XPS spectrum of Si $2 p$ and Si-C peaks

The Si $2 \mathrm{p}$ and the Si-C peaks were fitted by GaussianLorentzian $50-50 \%$ mixed functions, and the area of the peaks was calculated, which can be used to compare the amount of the carbon contamination of different treated fused silica surface. Comparing these ratios the highest $\mathrm{Si}-\mathrm{C} / \Sigma \mathrm{Si}$ ratios can be observed for naphthalene/methyl- 
methacrylate in the case of the highest concentration which means that the absorption coefficient is the highest. The ratio of carbon decreased with the lowering of concentration and the lowest $\mathrm{Si}-\mathrm{C} / \Sigma \mathrm{Si}$ ratio was observed for the pyrene/acetone solution having also the lowest absorption coefficient (Table 1).

\subsection{Ellipsometry}

The XPS detected carbon contamination suggested that the optical properties of the etched surface changed after the etching procedure, therefore the optical properties of the etched, completely cleaned fused silica substrates were determined in this study by Woollam M2000F rotating compensator spectroscopic ellipsometer. The ellipsometric angles $\Psi$ and $\Delta$ corresponding to the relative amplitude and phase change of the $s$ and $p$ polarized electric field vectors upon reflection, respectively, were measured at 4 different angles of incidence $\left(50^{\circ}, 55^{\circ}, 60^{\circ}\right.$ and $\left.65^{\circ}\right)$ and at 477 different wavelengths $(245-1000 \mathrm{~nm})$. In order to avoid the problems during data analysis arising from the light reflected from the sample backside, the fused silica backside was roughened. Upon reflection of the beam, the illuminated area was elliptical. The short axis was $1 \mathrm{~mm}$ and the long axis varies between $\sim 1.46 \mathrm{~mm}$ and $\sim 0.56 \mathrm{~mm}$, depending on the angle of incidence. Thus the illuminated area $\left(\sim 0.64 \mathrm{~mm}^{2}\right)$ was much smaller than the etched area $\left(15 \mathrm{~mm}^{2}\right)$.

The measured $\Psi$ and $\Delta$ curves belonging to the treated surface completely differ from the curves of the original surfaces (see Fig. 2.). This difference can be attributed to the carbon contamination.

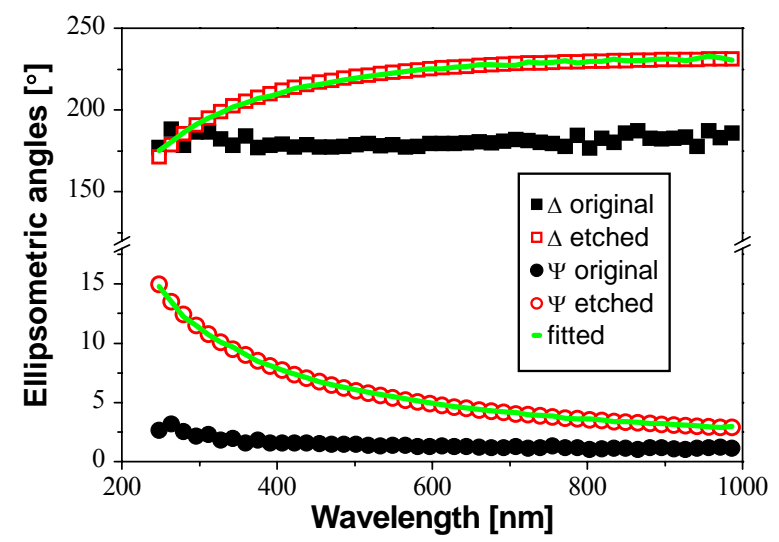

Fig. 2 The $\Psi$ (circles) and $\Delta$ (squares) values belonging to 55 angle of incidence measured on the unetched (original) quartz (solid symbols) and on the sample prepared at $500 \mathrm{~mJ} / \mathrm{cm}^{2}$ fluence (open symbols). For better visibility only every $10^{\text {th }}$ point is plotted. The solid line represents the result of the fitting with Cauchy's dispersion relation.

The optical properties of the original fused silica substrate were determined with Sellmeier's dispersion relation. Even though the surface roughness of the etched holes determined by AFM was below $5 \mathrm{~nm}$, as a first approach the etched holes were modeled as a roughened a quartz surface. For this a three phase model was applied, which consisted of the quartz substrate, surface roughness modeled with effective medium approximation [36] and ambient. The goodness of the fitting was measured by the difference of the fitted and measured $\Psi$ and $\Delta$ curves, namely with the Mean Squared Error, MSE [37]. The higher MSE values mean worse fitting. This roughness model resulted in MSE values exceeding 100 and thus it could not describe the measured data. Therefore the surface roughness layer was replaced in the three phase model with a fused silica layer of which refractive index and the extinction coefficient were described by Cauchy dispersion relation and Urbachtail, respectively. The average MSE of this type of fitting was 5.8, indicating good fittings and that the assumption of contaminated layer is plausible. The goodness of the fittings is represented also in Fig. 2, where the measured and fitted data is plotted (MSE=3.028). The Cauchy-Urbach model provide the optical properties of this carbon contaminated fused silica layer (at $248 \mathrm{~nm}$ ) produced by 1000 pulses of $\mathrm{KrF}$ laser (fluence: $500 \mathrm{~mJ} / \mathrm{cm}^{2}$ ) using naphthalene/methyl-methacrylate absorber $(c=1.71 \mathrm{M})$ : refractive index: $n=1.85$ (original fused silica: 1.51 ); absorption coefficient: $\alpha=1.58 \cdot 10^{5} \mathrm{~cm}^{-1}$ (absorption of original fused silica is negligible), while the thickness of the modified layer was measure to be $24 \mathrm{~nm}$ in this case. The increase of the refractive index and absorption coefficient can be due to the appearance of Si-C bonds (indicated by the XPS spectra).

The ellipsometrical measurements were carried out on several etched holes generated by $\mathrm{KrF}$ (and also $\mathrm{ArF}$ ) laser pulses in the fluence range of $480-1000 \mathrm{~mJ} / \mathrm{cm}^{2}$. Generally we state from these measurements that besides the slightly bonded deposited graphite-like layer [32-35] a modified, i.e. carbon contaminated, fused silica layer is present during LIBWE. The thicknesses of the modified, carbon contaminated fused silica surface layers are between 10 and $30 \mathrm{~nm}$ (KrF: 20-30 nm; ArF: 10-20 nm depending on the applied liquid absorber, concentration and fluence), the absorption coefficients are relatively high: measured at $248 \mathrm{~nm}$ for KrF etching: $1.0-1.8 \cdot 10^{5} \mathrm{~cm}^{-1}$, and measured at $193 \mathrm{~nm}$ for ArF etching: $2.5-4.0 \cdot 10^{5} \mathrm{~cm}^{-1}$, while the refractive index is increased from 1.51 to 1.85 (measured at $248 \mathrm{~nm}$ on $\mathrm{KrF}$ etched samples) according to our ellipsometric measurements.

\section{Numerical model and discussion}

Our previous numerical model [15] was completed according to our new results: the one dimensional heat flow equation was solved using the parameter of fused silica and considering the ellipsometry determined optical properties of the modified fused silica layer. The temporal intensity profile of the laser pulse was modeled by Gaussian profile. The melting and vaporization of the relevant materials were taken into account. Temperature-dependent material parameters (specific heat: $\mathrm{c}(\mathrm{T})$; heat diffusion coefficient: $\kappa(\mathrm{T}))$ were used in the available temperature ranges [15]. The absorption of the modified layer was also taken into account. Our previous model was further improved by considering the removal of the boiled fused silica: during these calculations, if the temperature of fused silica layer reached the boiling point, it leaved the surface, and the liquid absorber took its place - therefore the etch rate (which can be identify as the boiled fused silica) could be directly calculated with our program code. The elementary thickness of one layer was $\Delta \mathrm{z}=2.5 \mathrm{~nm}$, and the timestep was $\Delta \mathrm{t}=0.1 \mathrm{ps}$.

This improved model basically differs from our previously published theory [15] since we considered the presence of modified layer and the boiling of the fused silica 
during the laser pulse. If we took only the modified layer (without consideration of boiling) into account, the etch rate would increase (compared to our previous model); if we took only the boiling (without consideration of the modified layer) into account, the etch rate would slightly decrease (compared to our previous model). But using this two considerations in our new calculations, the improved model works for both laser wavelengths and gives relatively good results for the etch rates and threshold fluences.

The calculated and measured etch rates showed good agreement in the lower fluence range: for ArF: 200-400 $\mathrm{mJ} / \mathrm{cm}^{2}$; for $\mathrm{KrF}$ : 400-600 mJ/ $\mathrm{cm}^{2}$ (Fig. 3-4.).

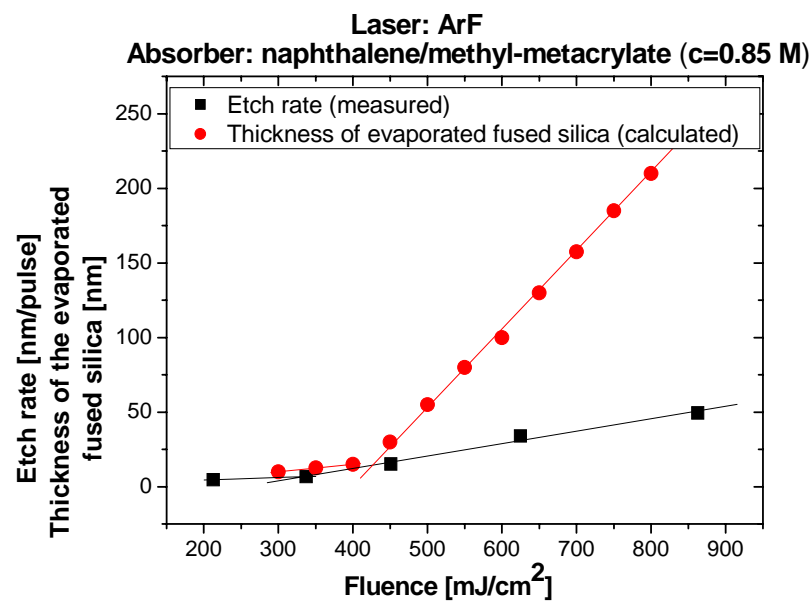

Fig. 3 The measured and calculated etch rate vs. fluence - for ArF laser (The thickness and the absorption coefficient of modified layer were $15 \mathrm{~nm}$ and $300000 \mathrm{~cm}^{-1}$ in this calculation, respectively.)

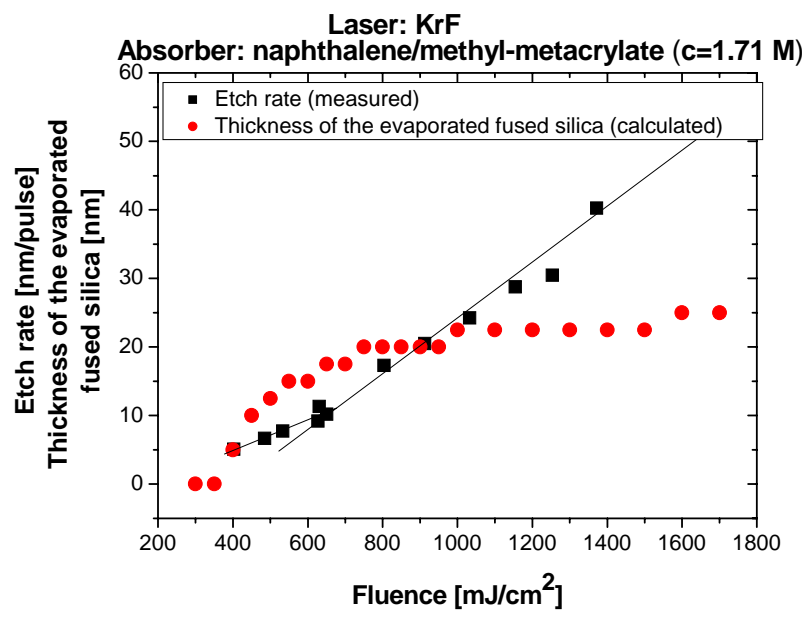

Fig. 4 The measured and calculated etch rate vs. fluence - for $\mathrm{KrF}$ laser (The thickness and the absorption coefficient of modified layer were $25 \mathrm{~nm}$ and $158000 \mathrm{~cm}^{-1}$ in this calculation, respectively.)

The thicknesses of the modified layers were determined directly from ellipsometric measurements, but the absorption coefficients at $193 \mathrm{~nm}$ were calculated from absorption measurements by spectrophotometer for the wavelength of ArF laser using the thickness values.

Our advanced model could predict the threshold fluences for both $\mathrm{ArF}$ and $\mathrm{KrF}$ etchings $\left(\mathrm{ArF}: \mathrm{F}_{\text {th }}{ }^{\text {meas } 193 \mathrm{~nm}}=210\right.$
$\mathrm{mJ} / \mathrm{cm}^{2} ; \quad F_{\text {th }}{ }^{\text {calc } 193 \mathrm{~nm}}=256 \mathrm{~mJ} / \mathrm{cm}^{2} ; \quad \mathrm{KrF}: \quad F_{\text {th }}{ }^{\text {meas } 248 \mathrm{~nm}}=400$ $\mathrm{mJ} / \mathrm{cm}^{2} ; F_{\text {th }}^{\text {calc } 248 \mathrm{~nm}}=395 \mathrm{~mJ} / \mathrm{cm}^{2}$ ), and describe the characteristic features of the experimentally determined fluence - etch rate graphs for ArF irradiation - the calculated fluence etch rate functions can be also fitted by two straight lines.

The breaking points on the measured fluence-etch rate graphs is at those fluence values (for $\mathrm{ArF}: 340 \mathrm{~mJ} / \mathrm{cm}^{2}$; for $\mathrm{KrF}: 620 \mathrm{~mJ} / \mathrm{cm}^{2}$ ), at which the modified layer can be completely removed already by one laser pulse. The measured etch rates at this fluences probably correspond to the thicknesses of the modified layers (for ArF: $7 \mathrm{~nm}$; for $\mathrm{KrF}$ : $10 \mathrm{~nm}$ ). If the applied fluences are higher than these, the slope of the function will be changed, because the modified layers are completely removed during the firs part of one pulse, and the etching mechanism is changed: only the organic liquid absorbs the further part of the pulse. The thickness of the residual melted layer was calculated to be 60-80 $\mathrm{nm}$ in the case of ArF laser; and 45-60 nm in the case of $\mathrm{KrF}$ laser. These layers can be identified as the modified layers, although these thicknesses were overestimated by the calculations. This residual melted and quickly refrozen layer might answer the question, how the modified layer is reproduced from pulse to pulse: the carbon (originate from the organic liquid molecules due to the high energy UV photons) probably can build in the upper part of the residual melted fused silica layer (by diffusion and/or due to the high pressure in the liquid [27, 29, 38]).

In the higher fluence region the difference between the measured and calculated etch rates became higher, although for using ArF laser the behavior of the fluence - etch rate graph is correct (namely the calculated data can also be fitted by two straight lines, similarly to our measurements).

This difference can be due to the weaknesses of the model, which were: 1 . the thermal and optical properties were not well known in the whole temperature and laser intensity ranges. Namely, the real material properties (c(T), $\kappa(\mathrm{T}), \alpha(\mathrm{I}))$ can differ from the used values. 2 . The graphitelike layer was not taken into account, because: i. it is difficult to characterize this layer (the model would be too speculative without correct characterization). ii. we can not say anything about the formation of this layer during the laser pulse (At the end of the etching procedure this layer can not be observed, but we have to suppose, that it has important role in the etching. Certainly the boiled and removed fused silica sweep away it from the surface.). 3 . The mechanical effects (high pressure bubbles [27, 29, 38]) were not included in the model.

\section{Summary}

To answer the open questions concerning the LIBWE procedure (two straight lines fitted fluence - etch rate graphs, modeling problems, and visual observations of graphite-like layer) two surface-analytical measurements (XPS and ellipsometry) were carried out. Our previous numerical model was developed according to these new results. On the basis of our experimental and numerical results, we gave a new LIBWE interpretation. We demonstrated that the described and characterized modified, carbon contaminated thin fused silica layer plays key role in the LIBWE procedure. 


\section{Acknowledgments}

The authors gratefully acknowledge the financial support of Hungarian Scientific Research Foundation (OTKA K67818 and CNK78549), and the Apponyi Albert Program of the National Office for Research and Technologies, Hungary (MEC-07-1-2009-0199).

\section{References}

[1] M. Bu, T. Melvin, G. J. Ensell, J. S. Wilkinson, A. G.R. Evans: Sensors and Actuators A 115 (2004) 476482

[2] E. Belloy, S. Thurre, E. Walckiers, A. Sayah, M.A.M Gijs: Sensors and Actuators 84 (2000) 330-337

[3] Ho-Su Jang, Myeong-Woo Cho and Dong-Sam Park: Sensors 8 (2008) 700-710

[4] S. Wang, C. Zhou, H. Ru, and Y. Zhang: Applied Optics 44 (2004) 4429-4432

[5] X. Lia, T. Abeb, M. Esashi: Sensors and Actuators A 87 (2001) 139-145

[6] Meng-Hua Yen, Ji-Yen Cheng, Cheng-Wey Wei, Yung-Chuan Chuang and Tai-Horng Young: J. Micromech. Microeng. 16 (2006) 1143-1153

[7] J. Ihlemann, S. Müller, S. Puschmann, D. Schäfer, M. Wei, J. Li, P.R. Herman: Appl. Phys. A 76 (2003) 751-753

[8] P.R. Herman, R.S. Marjoribanks, A. Oettl, K. Chen, I. Konovalov, S. Ness: Applied Surface Science 154-155 (2000) 577-586

[9] T. Makimura, S. Mitani, Youichi Kenmotsu, Kouichi Murakami, M. Mori. K. Kondo: Applied Physics Letters 85(7) 2004 1274-1276

[10] J. Ihlemann, B. Wolff, and P. Simon: Appl. Phys. A 54 (1992) 363-368

[11] S. I. Dolgaev, A. A. Lyalin, A. V. Simakin, G. A. Shafeev: Applied Surface Science 96-98 (1996) 491495

[12] J. Wang, H. Niino, A. Yabe: Appl. Phys. A 68 (1999)111-113

[13] R. Böhme, A. Braun, K. Zimmer: Applied Surface Science 186 (2002) 276-281

[14] G. Kopitkovas, T. Lippert, C. David, A. Wokaun, J. Gobrecht: Microelectronic Engineering 67-68 (2003) 438-444

[15] Cs. Vass, B. Hopp, T. Smausz, F. Ignácz: Thin Solid Films 453 -454 (2004) 121-126

[16] Ji-Yen Cheng, Meng-Hua Yen, Cheng-Wey Wei, Yung-Chuan Chuang and Tai-Horng Young: J. Micromech. Microeng. 15 (2005) 1147-1156
[17]Z.Q. Huang, M.H. Hong, T.B.M. Do, Q.Y. Lin: Appl Phys A 93 (2008) 159-163

[18] K. Fujito, T. Hashimoto, K. Samonji, J. S. Speck, S. Nakamura: Journal of Crystal Growth 272 (2004) 370 376

[19] B. Hopp, Cs. Vass, T. Smausz: Applied Surface Science 253 (2007) 7922-7925

[20] K. Zimmer, R. Böhme, B. Rauschenbach: Appl. Phys. A 79 (2004) 1883-1885

[21] J. Zhang, K. Sugioka, K. Midorikawa: Appl. Phys. A 67 (1998) 499-501

[22] J. Ihlemann: Applied Surface Science 54 (1992) 193200

[23] C. Vass, K. Osvay, B. Hopp, Z. Bor, Appl. Phys. A 87 (2007) 611-613

[24]C. Vass, K. Osvay, M. Csete, B. Hopp: Appl. Surf. Sci. 253 (2007) 8059-8063

[25] C. Vass, K. Osvay, B. Hopp, Opt. Express 14 (2006) 8354-8359

[26] C. Vass, 'K. Osvay, T. Véső, B. Hopp, Z. Bor: Appl Phys A 93 (2008) 69-73

[27] C. Vass, T. Smausz, B. Hopp: J. Phys. D Appl. Phys. 37 (2004) 2449-2454

[28] C. Vass, D. Sebők, B. Hopp: Applied Surface Science 252 (2006) 4768-4772

[29] X. Ding, Y. Kawaguchi, H. Niino, A. Yabe: Appl. Phys. A 75 (2002) 641-645

[30] G. Kopitkovas, T. Lippert, C. David, R. Sulcas, J. Hobley, A. Wokaun, J. Gobrecht: Proceedings of SPIE - The International Society for Optical Engineering 5662 (2004) 515-525

[31] G. Kopitkovas, T. Lippert, C. David, A. Wokaun, J. Gobrecht: Thin Solid Films 453-454 (2004) 31-35

[32] G. Kopitkovas, T. Lippert, C. David, S. Canulescu, A. Wokaun, J. Gobrecht: Journal of Photochemistry and Photobiology A: Chemistry 166 (2004) 135-140

[33] X.Ding, T. Sato, Y. Kawaguchi, H. Niino: Japanese Journal of Applied Physics Vol. 42 (2003) L176-L178

[34] R. Böhme, D. Spemann, K. Zimmer: Thin Solid Films 453-454 (2004) 127-132

[35] R. Böhme, Laser-induced backside wet etching of glasses and crystals, $\mathrm{PhD}$ thesis, Halle, 2006

[36] Handbook of Ellipsometry, ed: H. G. Tompkins, E. A. Irene, Springer, 2005

[37] I. Rajta, Sz. Szilasi, J. Budai, Z. Toth, P. Petrik, E. Baradacs: Nuclear Instruments and Methods in Physics Research Section B: Beam Interactions with Materials and Atoms 260(1) (2007) 400-404

[38] Y. Kawaguchi, X. Ding, A. Narazaki, T. Sato, H. Niino: Appl. Phys. A 79 (2004) 883-885

(Received: July 17, 2009, Accepted: January 13, 2010) 